\title{
Hidden Properties of Mathematical Physics Equations. Double Solutions. The Realization of Integrable Structures. Emergence of Physical Structures and Observable Formations
}

\section{I. Petrova}

Department of Computational Mathematics and Cybernetics, Moscow State University, Moscow, Russia Email: ptr@cs.msu.su

How to cite this paper: Petrova, L.I. (2020) Hidden Properties of Mathematical Physics Equations. Double Solutions. The Realization of Integrable Structures. Emergence of Physical Structures and Observable Formations. Journal of Applied Mathematics and Physics, 8, 1255-1262.

https://doi.org/10.4236/jamp.2020.87095

Received: May 27, 2020

Accepted: July 6, 2020

Published: July 9, 2020

Copyright (c) 2020 by author(s) and Scientific Research Publishing Inc.

This work is licensed under the Creative Commons Attribution International License (CC BY 4.0).

http://creativecommons.org/licenses/by/4.0/

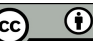

\section{Open Access}

\begin{abstract}
With the help of skew-symmetric differential forms the hidden properties of the mathematical physics equations are revealed. It is shown that the equations of mathematical physics can describe the emergence of various structures and formations such as waves, vortices, turbulent pulsations and others. Such properties of the mathematical physics equations, which are hidden (they appear only in the process of solving these equations), depend on the consistency of derivatives in partial differential equations and on the consistency of equations, if the equations of mathematical physics are a set of equations. This is due to the integrability of mathematical physics equations. It is shown that the equations of mathematical physics can have double solutions, namely, the solutions on the original coordinate space and the solutions on integrable structures that are realized discretely (due to any degrees of freedom). The transition from the solutions of the first type to one of the second type describes discrete transitions and the processes of origin of various structures and observable formations. Only mathematical physics equations, on what no additional conditions such as the integrability conditions are imposed, can possess such properties. The results of the present paper were obtained with the help of skew-symmetric differential forms.
\end{abstract}

\section{Keywords}

Integrability of Mathematical Physics Equations, Double Solutions, Integrable Structures, Discrete Transitions, Skew-Symmetric Differential Forms 


\section{Introduction}

We studied the equations of mathematical physics that are a set of equations and on which no additional conditions are imposed.

When studying the consistency of equations in system of equations, the evolutionary relation in skew-symmetric differential forms is obtained. The evolutionary relation obtained possesses a peculiarity, namely, it appears to be nonidentical one, which indicates the inconsistency of the equations.

From the nonidentity of evolutionary relation it follows that the mathematical physics equations have double solutions, namely, the solutions that are not functions (they depend not only on the variables, their derivatives be do not made up a differential) and solutions that are discrete functions. The specific feature consists in the fact that such solutions are defined on different spatial objects. The solutions of the first type are defined on the original coordinate space (their derivatives defined on a nonintegrable tangent manifold). And the solutions of the second type are defined on integrable structures. The transition from the solutions of the first type to one of the second type describes discrete transitions and the processes of origin of various structures and observable formations.

The results of the present paper were obtained with the help of skew-symmetric differential forms [1]. In this case, in addition to closed exterior skew-symmetric differential forms, which possess the invariant properties, it has been used the skew-symmetric differential forms, whose basis is non-integrable manifolds (as opposed to exterior skew-symmetric differential forms whose basis is integrable structures). These skew-symmetric forms, unlike closed exterior forms, are evolutionary ones. Such evolutionary forms (which contain an unconventional mathematical apparatus that includes such basic concepts as degenerate transformations and nonidentical relations) can describe evolutionary processes and generate closed exterior forms corresponding to invariant structures.

In the present paper, a study of the hidden properties of mathematical physics equations will be carried out by the example of equations that describe material media such as thermodynamic, gas-dynamic, cosmic media, systems of charged particles and so on.

It is known that the mathematical physics equations for material media consist of the equations of conservation laws for energy, linear momentum, angular momentum, and mass $[2,3]$, that is, they are a set of equations.

The integrability of such a set of differential equations depends on the consistency of equations in the set of equations.

\section{Analysis of Consistency of the Conservation Laws Equations. Evolutionary Relation for the State Functionals}

The peculiarity of present investigation consists in the fact that the conservation law equations are transformed into equations expressed in terms of state functionals [4]. The functionals such as wave function, entropy, the action functional and so on are functionals of the equations describing state material media. (Since the physical quan- 
tities such as temperature, energy, pressure, density relates to a single material medium, a connection between them should exist. The state functionals describe such a connection on which the state of the material medium depends.) ${ }^{1}$

Let us analyze the correlation of the equations that describe the conservation laws for energy and linear momentum. Consistency of equations can only be investigated using two coordinate systems.

We introduce two frames of reference: the first is an inertial one (this frame of reference is not connected with the material system), and the second is an accompanying one (this frame of reference is connected with the manifold built by the trajectories of the material medium elements).

In the accompanying frame of reference, that is connected with the manifold made up by the trajectories of material medium elements, the equation of energy and the equation for linear momentum may be written in the form

$$
\begin{gathered}
\frac{\partial \psi}{\partial \xi^{1}}=A_{1} \\
\frac{\partial \psi}{\partial \xi^{\nu}}=A_{\nu}, \quad \nu=2, \ldots
\end{gathered}
$$

Here $\psi$ are the state functional, $A_{1}$ and $A_{\nu}$ are the quantities that depends on specific features of material medium and accordingly on energy and force actions onto medium, $\xi^{1}$ and $\xi^{\nu}$ are the coordinates along the trajectory and in the direction normal to the trajectory. (In [5] Equations (1) and (2) are given for a gas-dynamic medium.)

Equations (1) and (2) can be convoluted into the relation

$$
d \psi=\omega
$$

where $\omega=A_{\mu} d \xi^{\mu}$ is the skew-symmetric differential form of the first degree [1].

In the general case (for energy, linear momentum, angular momentum and mass) this relation will be the form

$$
d \psi=\omega^{p}
$$

where $\omega^{p}$ is the skew-symmetric form of degree $p$ takes the values $p=0,1,2,3$.

The relations obtained and the skew-symmetric forms $\omega^{p}$ are evolutionary ones, since conservation laws equations are evolutionary ones.

The evolutionary relation obtained from the equations of conservation laws has, as will be shown, possesses unconventional properties. Such an evolutionary relation describes processes of emergence of various structures.

\section{Properties of Evolutionary Relation}

The evolutionary relation possesses nontraditional properties. This relation appears to be nonidentical and self-changed one. The skewsymmetric differential form, which is on the right-hand side, is not a closed form (its differential is not equal to zero) and cannot be a differential like the left-hand side. This relates to the fact that such

\footnotetext{
1 It can be noted that for a thermodynamic and gasdynamic media this state functional is entropy. But for a thermodynamic medium, this is entropy, which depend on thermodynamic variables, and for a gas-dynamic medium it is entropy, which depend on space-time coordinates.
} 
skew-symmetric form [1] (as opposed to exterior form which basis is an integrable manifold) is defined on an accompanying manifold, which is a deforming non-integrable manifold. The differential of such a form contains a differential of the manifold metric form, which specifies a manifold deformation and hence is nonzero.

(For example, the differential $d \omega$ of the form $\omega=A_{\mu} d \xi^{\mu}$ can be represented as $d \omega=K_{\alpha \beta} d \xi^{\alpha} d \xi^{\beta}$, where $K_{\alpha \beta}$ is the commutator of evolutionary form which can be written in the form (see Ref. [1]):

$$
K_{\alpha \beta}=\left(\frac{\partial A_{\beta}}{\partial \xi^{\alpha}}-\frac{\partial A_{\alpha}}{\partial \xi^{\beta}}\right)+\left(\Gamma_{\beta \alpha}^{\sigma}-\Gamma_{\alpha \beta}^{\sigma}\right) A_{\sigma}
$$

Here $\Gamma_{\beta \alpha}^{\sigma}$ is connectednesses of an accompanying manifold. The commutator $K_{\alpha \beta}$ is nonzero as the coefficients $A_{\mu}$ in real processes not potential (their mixed derivatives are not equal), and connectednesses of a accompanying manifold (which is a deforming non-integrable manifold) is not symmetric [6]. Thus it turns out that the commutator of the form $\omega$ and its differential does not equal to zero.) ${ }^{2}$

The non-identical evolutionary relation turns out to be self-changing one, that is, a change of one term leads to a change of another one and so on.

The non-identity of evolutionary relation, obtained from the equations of conservation laws, indicates that the conservation laws equations appear to be inconsistent. And this is due to the fact that the described system (in particular, the material medium) experiences non-potential effects.

The hidden properties of the equations of mathematical physics are connected precisely with the inconsistency of the equations that form the equations of mathematical physics.

\section{Double Solutions of the Equations of Mathematical Physics}

From the evolutionary relation it follows that the equations of mathematical physics can have double solutions, namely, the solutions, which are not functions (their derivatives do not made up a differential) and generalized solutions, i.e. solutions that are discrete functions.

Solutions to the mathematical physics equations on the original coordinate space.

The nonidentical evolutionary relation $d \psi=\omega^{p}$ cannot be integrated directly since its right-hand side contains unclosed skew-symmetric form which is not a differential. This means that the equations of mathematical physics prove to be non-integrable (they cannot be convoluted into identical relation for differentials and be integrated). In this case the solutions to equations of mathematical physics are not functions (their derivatives do not made up a differential). Such solutions will depend on the commutator of the evolutionary skewsymmetric form $\omega^{p}$ which is nonzero. (If the commutator be equal to zero, the evolutionary relation would be identical and this would point out to integrability of original equations.)

It turns out that solutions to the mathematical physics equations on the original coordinate space not a function. It depend on the commutator of the evolutionary skew-symmetric form $\omega^{p}$.

\footnotetext{
2 A concrete form of evolutionary relation for $p=1$ was considered for the Navier-Stokes equations in [5] for a gas-dynamic medium, and for electromagnetic field in the paper [1].
} 
Solutions to equations of mathematical physics on integrable structures.

It turns out that equations of mathematical physics can have solutions that are discrete functions.

From evolutionary relation it follows that under degenerate transformation, that does not conserve differential, from the evolutionary form (whose differential is not zero) the closed inexact (on a some integrable structure only) exterior form (whose differential is zero) and corresponding the closed dual form (describing the integrable structure) are realized.

The degenerate transformations can take place under additional conditions, which are due degrees of freedom. The vanishing of such functional expressions as determinants, Jacobians, Poisson's brackets, residues, and others corresponds to these additional conditions. The conditions of degenerate transformation can be realized under a change of nonidentical evolutionary relation, which, as it was noted, appears to be a self-varying relation.

The realization of closed inexact exterior form on a some integrable structure $\pi$ means that on integrable structure from nonidentical evolutionary relation $d \psi=\omega^{p}$ it follows the identical relation $d \psi_{\pi}=\omega_{\pi}^{p}$ , since the closed form $\omega_{\pi}^{p}$ is a differential.

Since the identical relation can be integrated (because it contains only of differentials), this means that on integrable structures the equations of mathematical physics become locally integrable only on integrable structures which is a pseudostructure with respect to its metric properties.

The solutions to the mathematical physics equations on integrable structures are generalized solutions, which are discrete functions, since they are realized only under additional conditions (on the integrable structures).

So, from the evolutionary relation it follows that the equations of mathematical physics can have double solutions.

But evolutionary relation has another unique property, which reveals the importance of double solutions to describe discrete transitions and processes of emergence of various structures and observed formations.

\section{Physical Meaning of Double Solutions}

Evolutionary relation $d \psi=\omega^{p}$ has an unique physical meaning because this relation includes a differential of a state functional $\psi$, which characterizes the state of material medium.

If from evolutionary relation the differential of a state functional could be obtained, this would point to the fact that state functional is a state function. And this would mean that the state of a material medium is a equilibrium one.

But, since evolutionary relation is a nonidentical relation, from that one cannot obtain the differential of state functional and find the state function. This means that the material medium is in a non-equilibrium state.

One can see that the solutions to the mathematical physics equations, which are not functions, describe a nonequilibrium state of material medium.

The nonequilibrium means that in the material medium an internal force acts. It is evident that the internal force is described by the commutator of skew-symmetric form $\omega$. (If the evolutionary form 
commutator be zero, the evolutionary relation would be identical, and this would point out to the equilibrium state, i.e. the absence of internal forces.) Everything that gives a contribution into the commutator of the evolutionary form $\omega$ leads to emergence of internal force that causes the non-equilibrium state of the material medium.

Evolutionary relation also describes a variation of non-equilibrium state. This is due to another peculiarity of nonidentical evolutionary relation, namely, this relation is a selfvarying relation.

As it has been shown above, under degenerate transformation the identical relation is obtained from nonidentical one.

From identical relation one can obtain the differential of state functional $d \psi$ and find state functional $\psi$ as a function of state. The availability function of state would point out to equilibrium state of the material medium. However, since the identical relation is satisfied only under additional conditions on integrable structures, such a state of the material medium will be a locally-equilibrium one.

One can see that the solutions to the mathematical physics equations, which are discrete functions, describe a locally-equilibrium state of material medium.

\section{Transition of Material Medium from Nonequilibrium State to Locally-Equilibrium State. Origination of Observable Formations and Physical Structures}

One can see that the transition from nonidentical relation to identical one points out to transition of material medium from non-equilibrium state into locally-equilibrium state.

As it has been shown above, the transition from nonidentical relation to identical points out to a transition from solutions of the first type to the generalized solutions.

It turns out that such transition is assigned to the transition of material medium from non-equilibrium state to locally-equilibrium state.

Since the non-equilibrium state has been induced by an availability of internal force and in the case of locally-equilibrium state there is no internal force (in local domain of material medium), it is evident that under transition of material medium from non-equilibrium state into locally-equilibrium state the nonmeasurable quantity, which acts as internal force, changes to a measurable quantity. This manifests itself in the form of arising a certain observable measurable formation. Waves, vortices, turbulent pulsations and so on are examples of such formations.

The generalized solutions to the equations of the mathematical physics describe such observable formations arisen.

It should be noted that closed dual forms and closed inexact exterior forms, which are realized under degenerate transformations, made up a differential-geometric structure, i.e. a integrable structure (pseudo structure) with conservative quantity (closed exterior form describes a conservative quantity because its differential equals zero). Realization of such differential-geometric structure (under degenerate transformation) points out to emergence of physical structure. The characteristics, the singular points, the envelopes of characteristics, and other 
structures with conserved quantities are examples of such physical structures.

\section{On the Problem of Numerical Solving the Equations of Mathematical Physics}

Problems of numerical solving the equations of mathematical physics relate to the fact that these solutions are defined on different spatial objects. The solutions of one type are defined on initial coordinate space whereas the solutions of another type are defined on integrable structures. Such solutions cannot be obtained by a continuous numerical simulation of derivatives.

The solutions of first type can be obtained only by numerical modeling the equations on the original nonintegrable manifold (it is impossible to find such a solution by analytical method). To obtain the generalized solutions by numerical simulation, one must use second systems of reference (on integrable structure). The generalized solutions can be obtaine also by analytical methods if the integrability conditions are imposed on the equations. The methods of characteristics, symmetries, eigen-functions and others are examples of such methods.

Therefore, to describe the emergence of observable formations and physical structures by numerical simulation, one must use two systems of reference, or by using simultaneously numerical and analytical methods.

\section{Conclusions}

It has been shown that the equations of mathematical physics can not only describe the change of physical quantities (such as energy, pressure, density), but also possess unique possibilities in describing evolutionary processes and the processes of emergence various structures and formations such as waves, vortices, turbulent pulsations and others.

Such properties of the equations of mathematical physics connected to the integrability of equations, which depends on consistency equations that form the equations of mathematical physics (and in particular depends on consistency derivatives according to different coordinates).

\section{Conflicts of Interest}

The author declares no conflicts of interest regarding the publication of this paper.

\section{References}

[1] Petrova, L.I. (2003) Exterior and Evolutionary Skew-Symmetric Differential Forms and Their Role in Mathematical Physics. http://arxiv.org/pdf/math-ph/0310050v1.pdf

[2] Clark, J.F. and Machesney, M. (1964) The Dynamics of Real Gases. Butterworths, London.

[3] Tolman, R.C. (1969) Relativity, Thermodynamics, and Cosmology. Clarendon Press, Oxford, UK. 
[4] Petrova, L.I. (2012) Physical Meaning and a Duality of Concepts of Wave Function, Action Functional, Entropy, the Pointing Vector, the Einstein Tensor. Journal of Mathematics Research, 4, 78-88.

https://doi.org/10.5539/jmr.v4n3p78

[5] Petrova, L.I. (2017) Features of Numerical Simulation of Euler and Navier-Stokes Equations. Computational Mathematics and Modeling, Consultants Bureau (United States), 28, 32-36. https://doi.org/10.1007/s10598-016-9343-0

[6] Tonnelat, M.A. (1959) Les principles de la theorie electromagnetique et la relativite. Masson, Paris. 